\title{
Product and Services Index
}

\section{A Guide to Microscopy Products}

\section{D Imaging and Measurement}

Xradia, Inc.

www.xradia.com

- MicroXCT'TM

- nanoXCT'M

\section{Aberration Corrected S/TEM}

JEOL USA $\quad$ www.jeolusa.com
- ARM200F

\section{Accessories (miscellaneous)}

Bel-Art Products

www.belart.com

McCrone Microscopes

www.mccronemicroscopes.com

\& Accessories

MTI Instruments/Fullam

www.mtiinstruments.com

p. 14

- Tensile/compression Testers

Spectronics Corporation www.spectroline.com

- UVG-50goggles+UVF-80 faceshield

XEI Scientific, Inc.

www.evactron.com

\section{Anti-vibration Devices}

Minus K Technology, Inc.

www.minusk.com

BM-1 Bench Top Vibration Isolator

- BM-4 Bench Top Vibration Isolator

- BM-10 Bench Top Vibration Isolator

- MK26 Vibration Isolation Workstation

- WS-4 Vibration Isolation Workstation

\section{Apertures (EM)}

\section{Electron Microscopy \\ Sciences}

See Company Profile

See Our Products

Ladd Research Industries

- TEM/SEM/FIB Apertures
215-412-8400

www.emsdiasum.com

p. 4

p. 10

\section{Atomic Force Microscopes (AFMs)}

A.P.E. Research SRL

- AFM A100-SGS

Advanced Research

Corporation

- MFM tip coatings

Agilent Technologies

- Agilent 5500 AFM

- Agilent 5420 AFM

- Agilent 5600LS AFM

- Agilent 6000ILM AFM (new)

AIST-NT

- SmartSPM

- CombiScope
Asylum Research

- MFP-3DTM-BIO

- MFP-3D ${ }^{\mathrm{TM}}$ Stand Alone

- Cypher ${ }^{\mathrm{TM}}$

- MFP Nanolndenter

\section{Bruker Nano}

- N8 NEOS

ION-TOF

- PPMS-AFM

Nanonics Imaging Ltd.

- The Hydra ${ }^{\top \mathrm{M}}$

Nanosurf AG

- easyScan 2 FlexAFM

- Nanite

Veeco Instruments, Inc.

- Dimension Icon AFM

- Bioscope Catalyst AFM
- easyScan 2 AFM

www.asylumresearch.com

www.bruker-nano.com

www.iontof.com

www.nanonics.co.il

www nanosurf.com

www.veeco.com

\section{Backscattered Electron Detectors (EM)}

Energy Beam Sciences, Inc. www.ebsstore.com

- Generation 5 Backscattered Electron Detector

\section{Beam Splitters}

Chroma Technology Corp.

www.chroma.com

Iridian Spectral Technologies www.iridian.ca

- Beam Splitters

Photometrics

www.photometrics.com

- Photometrics DV2

\section{Benchtop Electron Microscopes}

\section{DELONG America}

- LVEM5 Benchtop TEM/SEM/STEM

Www.LV-EM.com

\section{Biological Microscopes}

Meiji Techno America

www.meijitechno.com - MT5000

\section{Books}

McCrone Research Institute www.mcri.org

- Microscopy books, manuals, and texts

\section{Calibration}

Geller MicroAnalytical

www.GellerMicro.com

Lab., Inc.

- Elemental standards -UHV-EL

\section{Calibration Standards}

APPLIED IMAGE Inc.

www.appliedimage.com

- APPLIED SM, IAM, Accu-Place standards 


\section{Product and Services Index}

\section{A Guide to Microscopy Products}

\section{Calibration Standards (continued)}

Geller MicroAnalytical

www.GellerMicro.com

Lab., Inc.

- Magnification reference standards

Ted Pella, Inc.

- MetroChip SEM Calibration Standard

\section{Camera/Digital Camera Systems}

\section{Advanced Microscopy www.amtimaging.com}

Techniques Corp.

\section{JENOPTIK Optical}

www.jenoptik.com/progres

p. 13

\section{Systems, Inc.}

- ProgRes ${ }^{\circledR}$ microscope cameras

Lumenera Corporation www.lumenera.com

- Infinity USB 2.0 Digital Cameras

SIA (Scientific Instruments www.sia-cam.com and Applications)

\section{Cameras, CCD}

Advanced Microscopy

Techniques Corp.

Hamamatsu Corporation - ORCA Series Scientific CCD Cameras

\section{JENOPTIK Optical}

Systems, Inc.

- ProgRes ${ }^{\circledR}$ CCD cameras

Lumenera Corporation

- Infinity2

Photometrics

- Evolve

- CoolSnap HQ ${ }^{2}$

- CoolSnap EZ

- QuantEM:512SC

QImaging

- Exi Aqua

- Exi Blue

- QIClick

- QICAM Fast

- Retiga 2000R

\section{Cameras, CMOS}

Advanced Microscopy

Techniques Corp.

www.amtimaging.com

Hamamatsu Corporation www.sales.hamamatsu.com - ORCA Flash 2.8 Scientific CMOS cameras

\section{AXIOM OPTICS \\ www.axiomoptics.com}

- HICAM HighSpeed Intensified Camera

Lumenera Corporation

- Infinity1

\section{JENOPTIK Optical}

Systems, Inc.

- ProgRes ${ }^{\circledR}$ CMOS cameras

\section{Centrifuges}

PRO Scientific

www.proscientific.com

Chemicals

Ladd Research Industries

- Fixatives

- Stains etc for EM

- Light Microscopy

\section{Clean Room Accessories}

HEMCO Corporation

www.hemcoCorp.com

\section{Coating Custom}

Bel-Art Products

www.belart.com

\section{Coating Supplies (baskets, targets)}

Ted Pella, Inc.

www.tedpella.com

p. 16

- Evaporation Filaments, Boats

and baskets

\section{Coating Systems}

Denton Vacuum, LLC www.dantonvacuum.com

- Desk Top Pro

Energy Beam Sciences, Inc. www.ebsstore.com - Q150T Series Turbo Sputter Coaters

\section{Coating Thickness Gauge}

Kett US

- LZ370

www.kett.com

\section{Colloidal Gold}

\section{Electron Microscopy \\ Sciences \\ See Company Profile \\ See Our Products}

215-412-8400

www.emsdiasum.com

p. 4

p. 10

\section{Confocal Microscopes}

Harrick Scientific

www.harricksci.com

Products, Inc.

- TDIS Rheometer Accessory

Leica Microsystems

- Leica TCS STED CW

www.leica-microsystems.com p. 14

- Leica TCS SP5 II

- Leica TCS SPE

Olympus America, Inc. - LEXT OLS4000 ${ }^{\circledR}$ 


\section{Product and Services Index}

\section{A Guide to Microscopy Products}

\section{Confocal Microscopes (continued)}

Olympus America, Inc.

800-446-5967

www.olympusamerica.com

Olympus Soft Imaging

Solutions

See Company Profile

See Our Products
+49251798000

www.soft-imaging.net
McCrone Research Institute www.mcri.org

- Polarized light

- FTIR

- SEM

- Particle Handling Courses

\section{Critical Point Dryers}

p. 7

p. 14

\section{CrEST srl}

\section{Consulting}

\section{Alliance Technologies}

Particle Technology Labs

Polylnsight LLC

- Microscopy Services

\section{Contract Research}

Alliance Technologies

www.alliancetechgroup.com

McCrone Associates

Chemlmage Corporation

- Chemical Imaging Contract Services

Polylnsight LLC

www.polyinsight.com

- Microscopy Services

\section{Controllers (SPM)}

Specs Surface Nano

Analysis $\mathrm{GmbH}$

www.specs.com

- Nanonis control systems

\section{Correlative Microscopy}

\section{JEOL USA}

- ClairScope/JASM-6200

\section{Courses/Workshops}

College of Microscopy

www.collegeofmicroscopy.com

Lehigh Microscopy School www.lehigh.edu/microscopy

- Introduction to SEM and EDS for the New Operator

- Scanning Electron Microscopy and X-ray Microanalysis

- Scanning Probe Microscopy: From Fundamentals to Advanced Application

- Scanning Transmission Electron Microscopy: From Fundamentals to Advanced Applications

- Focused Ion Beam (FIB) Instrumentation and Applications

- Problem Solving with SEM

- X-ray Microanalysis

- Electron Backscatter Patterns

- Quantitative X-ray Microanalysis: Problem Solving Using EDS and WDS Techniques

\section{Electron Microscopy \\ Sciences}

See Company Profile

See Our Products
215-412-8400

www.emsdiasum.com

p. 4

Energy Beam Sciences, Inc.

www.ebsstore.com

- E3000/E3100 CPD Series

\section{Cryostats}

Janis Research Co.

- ST-500 Microscopy Cryostat

- ST-500-UC Ultra Compact Microscopy Cryostat

- CCS-100-XG-M Cryocooler for microscopy

\section{Cryotransfer Equipment (EM)}

Energy Beam Sciences, Inc. www.ebsstore.com - PP2000(T) Cryo-SEM

\section{Crystallographic Mapping}

Bruker Nano

www.bruker-nano.com

- QUANTAX CrystAlign

\section{Desiccators}

Geller MicroAnalytical

www.GellerMicro.com

Lab., Inc.

- VUS-J vacuum desiccators

\section{Detectors}

Bruker Nano

www.bruker-nano.com

p. 10

- QUANTAX

- QUANTAX CrystAlign

e2v Scientific Instruments www.e2vsi.com

- SiriusSD Silicon Drift Detector

p. 13

EDAX Inc.

800-535-edax

www.edax.com

\section{See Company Profile}

- Apollo X Silicon Drift Detector

- Apollo XL Silicon Drift Detector

- Hikari EBSD Detector

- DigiView IV EBSD Detector 


\section{Product and Services Index}

\section{A Guide to Microscopy Products}

\section{Detectors (continued)}

SII Nanotechnology USA, Inc. www.siintusa.com

- Detectors

- Silicon Drift Detectors

- 4 Element Detectors

- X-ray Detectors

\section{Devices}

Bel-Art Products

- Anti-Vibration

\section{Diamond Knives}

Delaware Diamond Knives www.ddk.com

- Diamond Knives, Histo Knives

\section{Diatome U.S.}

\section{5-412-8400}

www.emsdiasum.com

See Company Profile

See Our Products

Micro Star Technologies

www.microstartech.com

\section{Diamond Wire Saws}

Delaware Diamond Knives

www.ddk.com

- Diamond Wire Saw

South Bay Technology, Inc. www.southbaytech.com

- Diamond Wire Saw

Well Diamond Wire Saws, Inc. www.welldiamondwiresaws.com

- Saws Diamond Wire

\section{Digital Microscopes}

\section{CrEST srl}

Keyence Corporation

- VHX-1000 Digital Microscope

- VHX-500F Digital Microscope

LECO Corporation

\section{www.crestopt.com}

p. 10

www.keyence.com

www.leco.com p. 2

p. 10

\section{Dual Beam FIB/SEM}

Hitachi

- NB5000

Nanonics Imaging Ltd.

Tescan USA Inc.

- VELA-3

- LYRA-3

\section{E-Beam Lithography}

SEMTech Solutions, Inc. 978-663-9822 (235)

\section{www.semtechsolutions.com}

See Company Profile

- Electron Beam Lithography Systems

- Add-ons for SEMs

Tescan USA Inc.

www.tescan.com

- DrawBeam

\section{EDS Detector Repairs/Upgrades}

4pi Analysis, Inc. www.4pi.com

e2v Scientific Instruments www.e2vsi.com

- Si(Li) EDS Detector Repairs

MAX Detector Repair $\quad$ www.maxdetector.com

Group LLC

- Liquid Nitrogen X-Ray Detector Repair+Service

\section{Electron Backscatter Diffraction (EBSD)}

Bruker Nano

www.bruker-nano.com

p. 10

-QUANTAX CrystAlign

\section{EDAX Inc.}

See Company Profile

- OIM ${ }^{\mathrm{TM} A n a l y s i s ~ S y s t e m}$

- Hikari Detector

- DigiView IV Detector

\section{Oxford Instruments}

NanoAnalysis

- HKL Nordlys Detectors

\section{0-535-edax}

\section{www.edax.com}

p. 3

\section{Electron Beam Microjoining Machine}

Seron Technologies Inc.

www.serontech.co.kr - AIW 100

\section{Electron Microprobe Automation}

Advanced MicroBeam, Inc. www.advancedmicrobeam.com

Geller MicroAnalytical Lab., www.GellerMicro.com Inc.

- dQant electron probe automation system

\section{Electron Microprobe Service}

Advanced MicroBeam, Inc. www.advancedmicrobeam.com

\section{Electron Microprobes (EPMAs)}

Advanced MicroBeam, Inc.

www.advancedmicrobeam.com 


\section{Product and Services Index}

\section{A Guide to Microscopy Products}

\section{Electron Multiplying CCD (EMCCD)}

Hamamatsu Corporation www.sales.hamamatsu.com

- ImagEM series EMCCD Cameras

\section{Elements and Compounds (fine chemicals)}

Kurt J. Lesker Co.

www.lesker.com

- Sputtering materials \& targets

\section{Embedding Materials}

\section{Electron Microscopy}

Sciences

See Company Profile

See Our Products

Tousimis Research

Corporation

- TEM Embedding Resins

\section{5-412-8400}

www.emsdiasum.com

\section{Energy-Dispersive X-ray Spectrometry (EDS)}

4pi Analysis, Inc.

www.4pi.com

p. 10

Bruker Nano

www.bruker-nano.com

p. 10

- QUANTAX

e2v Scientific Instruments www.e2vsi.com

- SDD and Si(Li) EDS Detectors

- DXPs

\section{EDAX Inc.}

800-535-edax www.edax.com

\section{See Company Profile}

- TEAM ${ }^{\mathrm{TM}}$ Analysis System

- Genesis Analysis System

- Apollo X Silicon Drift Detector

- Apollo XL Silicon Drift Detector

\section{Evex Inc.}

\section{9-252-9192}

\section{www.evex.com}

\section{See Company Profile}

\section{Oxford Instruments}

NanoAnalysis

- X-Max

- INCA Energy

\section{Environmental Chambers}

\section{Electron Microscopy \\ Sciences}

See Company Profile

See Our Products
215-412-8400

www.emsdiasum.com

\section{Failure Analysis}

Advanced Research

Corporation

- Analytical Services

Gatan, Inc.

- Gatan Centar Frontier

McCrone Associates

Polylnsight LLC

- Microscopy Services

www.arcnano.com

www.gatan.com

www.mccroneassociates.com

www.polyinsight.com

\section{FIB Accessories}

Advanced Research

Corporation

- FIB Apertures Custom OEM

Nanonics Imaging Ltd. $\quad$ www.nanonics.co.il p. 14

- SPM NSOM Integrated with FIB

Omniprobe, Inc. www.omniprobe.com

- AutoProbe ${ }^{\circledR}$

Ted Pella, Inc.

www.tedpella.com

p. 16

- FIB Grids

- Needles

- Holders

www.arcnano.com

\section{Fiber Optic Illuminators}

X-Cite by EXFO

www.exfo-xcite.com

p. 16

- X-Cite exacte

- Liquid Light Guides

Sutter Instrument

www.sutter.com

p. 15

\section{Field Emission SEMs (FESEMs)}

Tescan USA Inc.

www.tescan.com

p. 16

- MIRA-3

\section{Filaments and Filament Rebuilding}

\section{Electron Microscopy}

Sciences

See Company Profile

See Our Products
215-412-8400

www.emsdiasum.com

p. 4

p. 10

M.E. Taylor Engineering, Inc. www.semsupplies.com - Filaments

Ted Pella, Inc.

www.tedpella.com

- Tungsten

- $\mathrm{LaB}_{6}$ and Field Emission EM Filaments

\section{Filters}




\section{Product and Services Index}

\section{A Guide to Microscopy Products}

\section{Filter Switching}

Sutter Instrument

- Lambda 10-3

\section{Fixatives and Chemicals}

SPI Supplies

- SPI-Chem

Tousimis Research

Corporation

- Ultrapure Chemicals

\section{Fluorescence Analysis}

Spectronics Corporation www.spectroline.com

- CM-10MP mini UV viewing workstation

\section{Fluorescence Microscopy}

AXIOM OPTICS

www.axiomoptics.com

- LIFA Frequency Domain FLIM

Chroma Technology Corp.

www.chroma.com

\section{CrEST srl}

\section{www.crestopt.com}

www.exfo-xcite.com

p. 10

X-Cite by EXFO

- X-Cite exacte

Keyence Corporation

www.keyence.com

- BZ-9000 All-in-one Flourescence Microscope

Lambert Instruments

www.lambert-instruments.com

- LIFA:Lambert Instruments FLIM attachment

Lumenera Corporation

www.lumenera.com

- Infinity 3-10

\section{Media Cybernetics}

www.mediacy.com

p. 14

MICROS Produktions- und www.micros.at

Handels $\mathrm{GmbH}$

- Lily MCX500

Navitar, Inc.

- 2FL Video Fluorescence Scope

Renishaw, Inc. www.renishaw.com

- Raman Microscope System

Special Optics

www.specialoptics.com

- Custom Lenses

Sutter Instrument

Ted Pella, Inc.

\section{www.sutter.com}

www.tedpella.com

- The PELCO BioWave Pro Microwave Tissue Processor

\section{Focused Ion Beam Systems}

Carl Zeiss SMT Inc.

- AURIGA ${ }^{\circledR}$ FIB-SEM

JEOL USA

- MultiBeam:JIB-4500/4600F

\section{Forensic Testing Supplies}

Spectronics Corporation www.spectroline.com

- OFK-7000 Optimax ${ }^{T M}$ Multi-Lite ${ }^{T M}$ LED Forensic Light Source Kit

Forster Resonance Energy Transfer (FRET) Microscop

Lambert Instruments

www.lambert-instruments.com

- II18MDS:Component for FLIM systems

\section{Freeze Drying Equipment}

McCrone Microscopes

www.mccronemicroscopes.com

\& Accessories

\section{Friction Analyzer}

Kett US $\quad$ www.kett.com
- H94ai

\section{FT-IR Microscopy}

Alliance Technologies

Renishaw, Inc.

- Raman Microscope System

\section{Gas Injection Systems}

Omniprobe, Inc.

- OmniGIS ${ }^{\circledR}$

\section{Grids (EM)}

Dune Sciences, Inc.

- SMART GRIDS - Nanogrids ${ }^{T M}$

- SMART GRIDS - Biogrids' ${ }^{\text {TM }}$

\section{Electron Microscopy}

Sciences

215-412-8400

See Company Profile www.emsdiasum.com

www.alliancetechgroup.com

www.renishaw.com

www.omniprobe.com

WwW.dunesciences.com

See Our Products

\section{Heating Stages}

ibidi, LLC

www.ibidi.com

- Heated Stage- 96 Well Format

\section{Helium Ion Microscope}

Carl Zeiss SMT Inc. - ORION ${ }^{\circledR}$

www.zeiss.com/nts

\section{High Pressure Freezing}

Leica Microsystems

www.leica-microsystems.com p. 14

- Leica EM PACT2

- Leica HPM 


\section{Product and Services Index}

\section{A Guide to Microscopy Products}

\section{Histology Supplies}

Globe Scientific Inc.

- Slides

\section{Illuminators (LOM)}

CoolLED Ltd.

www.coolled.com

p. 10

- pE-series LED illumination products

PRO Scientific

www.proscientific.com

Sutter Instrument

www.sutter.com

- Lambda XL

X-Cite by EXFO

- X-Cite exacte

- X-Cite 120 Series

\section{Image Analysis}

Amerinex Applied Imaging

www.amerineximaging.com

- Aphelion

Buehler

- OmniMet Digital Imaging Software

\section{JENOPTIK Optical}

www.jenoptik.com/progres

Systems, Inc.

- ProgRes ${ }^{\circledR}$ microscope cameras

- CapturePro Software

Olympus America, Inc.

- CellSens ${ }^{\mathrm{TM}}$ Software

- Stream Software

\section{Olympus America, Inc.}

800-446-5967

www.olympusamerica.com

\section{Olympus Soft Imaging}

Solutions

\section{+49251798000 \\ www.soft-imaging.net}

\section{See Company Profile}

See Our Products

Particle Technology Labs

www.particletechlabs.com

Ted Pella, Inc.

www.tedpella.com

- The PELCO BioWave Pro Microwave Tissue Processor

\section{Image Analysis Software}

Media Cybernetics

www.mediacy.com

p. 14

- Image-Pro Plus Software

Olympus America, Inc.

800-446-5967

www.olympusamerica.com

Olympus Soft Imaging

Solutions

+49251798000

www.soft-imaging.net

\section{See Company Profile}

See Our Products

\section{p. 7}

p. 14

\section{Image Analyzers}

Fluid Imaging Technologies, www.fluidimaging.com Inc.

- Benchtop Flowcam ${ }^{\circledR}$

- Submersible Flowcam ${ }^{\circledR}$

- PETROCAM ${ }^{\circledR}$

- Portable Flowcam ${ }^{\circledR}$

\section{Image Processing}

Amerinex Applied Imaging - Aphelion

AXIOM OPTICS

- Adaptive Optics Kit Bio

HREM Research Inc.

- Deconvolution (DeConvEELS/DeConvHAADF)

- Software Cs-correction (FTSR/IWFR)

- Strain Analysis (GPA/PPA)

\section{JENOPTIK Optical}

www.jenoptik.com/progres

Systems, Inc.

- ProgRes ${ }^{\circledR}$ microscope cameras

- CapturePro Software

Lumenera Corporation

www.lumenera.com

- Infinity Analyze

\section{Image Processing Software}

\section{Media Cybernetics \\ - AutoQuant Software}

www.mediacy.com

p. 14

\section{Incubators for Live Cell Imaging}

ibidi, LLC

www.ibidi.com

- Heating+Incubation Unit for Microscope Stage

\section{Indenters}

Micro Star Technologies

www.microstartech.com

\section{Infra-Red Microscopes}

CRAIC Technologies, Inc. - UVM-1NIR Microscope

www.microspectra.com

\section{Ion Beam Milling}

$\begin{array}{lrr}\begin{array}{l}\text { Hitachi } \\ \text { - NB5000 }\end{array} & \text { www.hitachi-hta.com } & \text { p. } 12 \\ \begin{array}{l}\text { Leica Microsystems } \\ \text { - Leica RES101 }\end{array} & \text { www.leica-microsystems.com } & \text { p. } 14 \\ \begin{array}{l}\text { South Bay Technology, Inc. } \\ \text { - Low Energy, Low Angle, Large Area lon Beam Polisher }\end{array} & \end{array}$

\section{Ion Guns}

www.lesker.com
Kurt J. Lesker Co.

- Ion sources

- Power supplies 


\section{Product and Services Index}

\section{A Guide to Microscopy Products}

\section{Ion Pumps, New and Rebuilt}

Gamma Vacuum

- Ion Pumps

- Ion Pump Controllers

\section{Journals}

McCrone Research Institute www.mcri.org

- The Microscope Journal

\section{Knife Resharpening}

\section{Diatome U.S.}

215-412-8400

www.emsdiasum.com

\section{See Company Profile}

See Our Products

p. 2

p. 10

\section{Knife Sharpening}

Delaware Diamond Knives www.ddk.com
- Tungsten Carbide, Sapphire, Diamond Knives

Micro Star Technologies www.microstartech.com

\section{Knives}

\section{Diatome U.S.}

215-412-8400

\section{www.emsdiasum.com}

\section{See Company Profile}

See Our Products

p. 2

p. 10

Minitool, Inc.

www.minitoollnc.com

- Minitool Precision MicroTools

\section{$\mathrm{LaB}_{6}$ Sources}

\section{Electron Microscopy \\ Sciences}

See Company Profile

See Our Products

\section{www.emsdiasum.com}

p. 4

p. 10

\section{Laboratory Information Management System (LIMS)}

Zumatrix, Inc.

www.zumatrix.com

- Matrix Gemini LIMS

- Matrix Express LIMS

- Matrix Track-IT- Sample Tracking

- Matrix Stability-Stability Study Management

\section{Laboratory Safety Supplies}

HEMCO Corporation

www.hemcoCorp.com

\section{Laminar Flow Hoods}

\section{Lamps and Light Sources}

CoolLED Ltd.

www.coolled.com

- $p E$-series LED illumination products

Spectronics Corporation www.spectroline.com

- E-Series + X-Series Longwave lamps

\section{Sutter Instrument}

www.sutter.com

- Lambda XL

\section{Lasers}

COBOLT AB

\section{www.cobolt.se}

- Cobolt Calypso'M $401 \mathrm{~nm}$ DPSS

- Cobolt Jive ${ }^{\mathrm{TM}} 561 \mathrm{~nm}$ DPSS

- Cobolt Zouk ${ }^{\mathrm{TM}} 355 \mathrm{~nm}$ CW

- Cobolt Mambo'TM 594 nm DPSS

- Cobolt Fandango ${ }^{\mathrm{TM}} 515$ nm DPSS

Renishaw, Inc.

www.renishaw.com

- Raman Microscope System

\section{Laser Scanning Confocal Microscopes}

Keyence Corporation www.keyence.com

- VK-9700 Laser Scanning Confocal Microscope

Olympus America, Inc. $\quad$ www.olympusamerica.com $\quad$ p. 14

- FluoView FV10i

Olympus America, Inc.

\section{0-446-5967}

www.olympusamerica.com

Olympus Soft Imaging

+49251798000

Solutions

www.soft-imaging.net

\section{See Company Profile}

See Our Products

p. 7

\section{Leak Detection}

Pfeiffer Vacuum

www.pfeiffer-vacuum.com

\section{LED}

Prizmatix

www.prizmatix.com

- Fiber Coupled LED Light Sources

\section{LED Illumination (LOM)}

CoolLED Ltd.

www.coolled.com

p. 10

- $p E$-series LED illumination products

Prizmatix

- Ultra-High Power LED Light Source

- UV High Power LED Light Source

- Modular Multi-Wavelength LED Light Source

\section{LED Lamps}

CoolLED Ltd

www.coolled.com

p. 10 


\section{Product and Services Index}

\section{A Guide to Microscopy Products}

\section{Lenses (LOM)}

Navitar, Inc.

- High Mag Imaging Lenses

Special Optics www.specialoptics.com

- Confocal Microscopy Laser Scanning Lenses

\section{Life Sciences}

\section{Media Cybernetics}

www.mediacy.com

Ted Pella, Inc. www.tedpella.com

- High resolution FE-SEM Sputter Coaters

- The PELCO BioWave Pro Microwave Tissue Processor

- PELCO easiGlow Grid Cleaning System

\section{Light Optical Microscopes (LOMs)}

\section{Bruker Nano}

www.bruker-nano.com

p. 10

- N8 NEOS

MICROS Produktions- und

www.micros.at

Handels GmbH

- Crocus II MCX100LCD

Mitutoyo America Corp.

www.mitutoyo.com

\section{Live Cell Chambers}

Charles Supper Company, Inc. www.charles-supper.com

- MVD/24 Crystal Growth Chambers

\section{Live-Cell Imaging}

\section{CrEST srl}

ibidi, LLC

- ibidi perfusion System

- U-slide Chemotaxis

Lambert Instruments

- Li2Cam MDS: component for FLIM systems

Nanonics Imaging Ltd. Www.nanonics.co.il

- The Hydra ${ }^{\mathrm{TM}}$

\section{Low Voltage Electron Microscopes (LVEM)}

\section{DELONG America}

- LVEM5 Benchtop TEM/SEM/STEM

\section{Magnetic Field Cancellation}

\section{Technical Manufacturing www.techmfg.com}

Corporation

- Mag-NetX'TM Magnetic Field Cancellation System

\section{Magnification Calibration}

\section{Material Science}

Ted Pella, Inc.

www.tedpella.com

- High resolution FE-SEM Sputter Coaters

- PELCO easiGlow Grid Cleaning System

\section{Measuring Microscopes}

Zemetrics, Inc.

www.zemetrics.com

p. $17 \stackrel{0}{0}$

- ZeMapper 3D Metrology System

Zygo Corporation www.zygo.com

\section{Mercury Lamps}

\section{X-Cite by EXFO}

- X-Cite exacte

www.exfo-xcite.com

Metal Halide Lamps

\section{X-Cite by EXFO}

www.exfo-xcite.com

p. 16

- X-Cite exacte

- X-Cite lamps

\section{Metallographic Equipment}

\begin{tabular}{|c|c|}
\hline Allied High Tech Products & www.alliedhightech.com \\
\hline $\begin{array}{l}\text { Buehler } \\
\text { - IsoMet } 4000 \text { Linear Precisior }\end{array}$ & $\begin{array}{l}\text { www.buehler.com } \\
\text { Saw }\end{array}$ \\
\hline LECO Corporation & www.leco.com \\
\hline
\end{tabular}

Oxford Instruments www.oxford-instruments.com p. 15

NanoAnalysis

- HKL Nordlys Detectors

\section{Metallographic Microscopes}

Allied High Tech Products www.alliedhightech.com

\section{Metallurgical Microscopes}

\section{LECO Corporation \\ Meiji Techno America - MT7000}

MICROS Produktions- und

Handels $\mathrm{GmbH}$

- Platinum MCXM500

\section{Metrology Instruments}

AXIOM OPTICS

- HASO32 Wavefront Sensor

Charles Supper Company, Inc. www.charles-supper.com

- Supper Spindle Stage

Hysitron, Inc.

- Hysitron Ubi 750

MTI Instruments/Fullam www.mtiinstruments.com

- Tensile/Compression Testers www.leco.com

www.meijitechno.com

www.micros.at

www.axiomoptics.com

www.hysitron.com

p. 13

p. 14 


\section{Product and Services Index}

\section{A Guide to Microscopy Products}

\section{Metrology Instruments (continued)}

\section{Oxford Instruments}

NanoAnalysis

- ThinFilmID

Zygo Corporation

www.zygo.com

\section{Microanalysis Services}

\section{Advanced MicroBeam, Inc. www.advancedmicrobeam.com \\ McCrone Associates www.mccroneassociates.com}

\section{Microinjectors}

Applied Scientific

Instrumentation (ASI)

- MPPI-3

\section{Sutter Instrument}

www.sutter.com

p. 15

\section{Micromanipulators}

Janis Research Co.

- ST-500 Low Vibration Probe Station

Minitool, Inc.

www.minitoollnc.com

- Minitool Model FX-117

www.omniprobe.com

Omniprobe, Inc.

- ValueProbe ${ }^{\mathrm{TM}}$

Sutter Instrument
Zemetrics, Inc.

- ZeMapper 3D Metrology System

- MPC-385 / MPC-325

\section{Microscope Viewing Heads}

Charles Supper Company, Inc. www.charles-supper.com

- Specimen Mounting Heads

Haydon Kerk Motion

www.haydonkerk.com

Solutions, Inc.

Microscopy Services

McCrone Associates

www.mccroneassociates.com

SEMTech Solutions, Inc. 978-663-9822 (235)

www.semtechsolutions.com

See Company Profile

- SEM Anayltical Lab Services

Zygo Corporation

www.zygo.com

\section{Microscopy Training}

College of Microscopy

www.collegeofmicroscopy.com

\section{Microtomes and Ultramicrotomes}

MICROS Produktions-

www.micros.at

und Handels GgmbH

- Microstome Razor and Steely

\section{Microwaves}

\section{Electron Microscopy}

215-412-8400
Chroma Technology Corp.

Globe Scientific Inc.

Haydon Kerk Motion

Solutions, Inc.

\section{Microscope Stages}

Haydon Kerk Motion

Solutions, Inc.

McCrone Microscopes

\& Accessories

Sutter Instrument
- Microscope Slides www.haydonkerk.com

www.mccronemicroscopes.com

www.sutter.com

p. 15

\section{Microscope Stands}

Sciences

See Company Profile

p. 4

See Our Products

\section{Microwave Processing}

\section{www.emsdiasum.com}

Ted Pella, Inc. - PELCO BioWave Pro

www.tedpella.com

p. 16

\section{Moisture Balance}

Kett US

- FD720

www.kett.com

\section{Multiprobe Imaging}

Nanonics Imaging Ltd.

- The Hydra ${ }^{\mathrm{TM}}$

www.nanonics.co.i

p. 14

\section{Nanoindentation}

Hysitron, Inc.

www.hysitron.com

p. 13

- Tribolndenter TI 950

p. 14
Nanofactory Instruments AB www.nanofactory.com

- In situ TEM Systems 


\section{Product and Services Index}

\section{A Guide to Microscopy Products}

\section{Nanopositioners}

Charles Supper Company, Inc. www.charles-supper.com

- Micropositioners

Nanonics Imaging Ltd. $\quad$ www.nanonics.co.il

- The Nanonics MultiView AFM-Raman Systems

\section{Nanoprobes}

Advanced Diamond

www.thindiamond.com

Techonologies

- NaDiaProbes-All Diamond AFM Probes

\section{Evex Inc.}

609-252-9192

www.evex.com

See Company Profile

p. 5

Micro Star Technologies

www.microstartech.com

Nanofactory Instruments AB www.nanofactory.com - In situ TEM Systems

\section{Near-Field Scanning Optical Microscopes (NSOMs)}

A.P.E. Research SRL

www.aperesearch.com

- TriA-SNOM

Nanonics Imaging Ltd.

www.nanonics.co.il

p. 14

- Near-Field Scanning Optical Microscopes

- The HydraTM

\section{NIR Composition}

Kett US

- KJT270

\section{NIR Moisture Meter}

Kett US

- KJT130

\section{Objective Lenses}

Mitutoyo America Corp.

www.mitutoyo.com

Special Optics

- Laser Focusing Objectives

\section{Optical Filters}

Chroma Technology Corp.

www.chroma.com

Iridian Spectral Technologies www.iridian.ca

- Optical Filters

- Raman Optical Filters

- Fluorescence Optical Filters

\section{Optical Power Measurement}

X-Cite by EXFO

www.exfo-xcite.com

p. 16

- X-Cite Optical Power Measurement System

\section{Optical Prototyping System}

Prizmatix

www.prizmatix.com

- OptiBlock Optical Prototyping System

\section{Phase Contrast Microscopy}

HREM Research Inc.

www.hremresearch.com

- QPt for DigitalMicrograph

Photography Supplies

$\begin{array}{ll}\text { Electron Microscopy } & \mathbf{2 1 5 - 4 1 2 - 8 4 0 0} \\ \text { Sciences } & \text { www.emsdiasum.com }\end{array}$

See Company Profile

See Our Products

p. 4

p. 10

\section{Plasma Cleaners}

Gatan, Inc.

- Gatan 950 Solarus ${ }^{\circledR}$

www.gatan.com

p. 11

South Bay Technology, Inc. www.southbaytech.com

- Large Capacity Plasma Cleaner

XEI Scientific, Inc.

www.evactron.com

p. 16

\section{Polarized Light Microscopy}

McCrone Microscopes

\& Accessories

Meiji Techno America - MT9900

\section{Polishing Consumables}

Allied High Tech Products

Buehler

- Buehler SunMet Polishing Bundles

www.mccronemicroscopes.com

www.meijitechno.com

Polishing Equipment

Allied High Tech Products

www.alliedhightech.com

p. 10

LECO Corporation

www.leco.com

South Bay Technology, Inc. www.southbaytech.com

- Polishing Machines and Fixtures

\section{Raman Spectroscopy/Microscopy}

AIST-NT

www.aist-nt.com

- OmegaScope

Chemlmage Corporation www.chemimage.com

- Falcon II Wide-Field Raman Chemical Imaging System 


\section{Product and Services Index}

\section{A Guide to Microscopy Products}

\section{Raman Spectroscopy/Microscopy (continued)}

Harrick Scientific

www.harricksci.com

Products, Inc.

- Flat-Top Environmental Chamber Accessory

Nanonics Imaging Ltd. www.nanonics.co.il

- The Nanonics MultiView AFM-Raman Systems

- SPM/TERS/NSOM Integrated with Raman Spectroscopy

Renishaw, Inc.

www.renishaw.com

- Raman Microscope System

\section{Reflection Light Microscope}

Capovani Brothers Inc.

www.capovani.com

- Leitz Ergolux Reflected Light Microscopes

\section{Resharpening Services}

Diatome U.S.

\section{See Company Profile}

See Our Products

\section{5-412-8400}

www.emsdiasum.com

p. 2

p. 10

\section{Residual Gas Analyzers}

Pfeiffer Vacuum

- Mass Spec

www.pfeiffer-vacuum.com

\section{Resolution Standards}

APPLIED IMAGE Inc.

www.appliedimage.com

- APPLIED Resolution charts and arrays

Geller MicroAnalytical Lab., www.GellerMicro.com Inc.

- MRS-5

\section{Sample Preparation and Handling}

Buehler

www.buehler.com

- \#63-2000 Buehler Vanguard Fully Automatic Preparation System

Denton Vacuum, LLC

www.dantonvacuum.com

- DV-502B

\section{Diatome U.S.}

\section{See Company Profile See Our Products}

\section{5-412-8400} www.emsdiasum.com

p. 2

p. 10

Dune Sciences, Inc. www.dunesciences.com

- SMARD GRID Sample Preparation Videos

\section{Electron Microscopy Sciences}

See Company Profile See Our Products

\section{5-412-8400} www.emsdiasum.com p. 4

p. 10
Minitool, Inc.

www.minitoollnc.com

- Minitool 55-0078 MicroRuler

- Minitool Precision MicroTools

PRO Scientific

www.proscientific.com

South Bay Technology, Inc.

- Sample Preparation Tools

Ted Pella, Inc.

- PELCO SEM mounts and SEM mount storage boxes

\section{Scanners (SPM)}

Nanonics Imaging Ltd.

www.nanonics.co.il

- The Nanonics MultiView AFM-Raman Systems

\section{Scanning Electron Microscopes (SEMs)}

Carl Zeiss SMT Inc.

- MERLIN ${ }^{\circledR}$ FE-SEM

DELONG America

- LVEM5 Benchtop TEM/SEM/STEM

\section{Evex Inc.}

609-252-9192

\section{www.evex.com}

See Company Profile

FEI

- Magellan XHR

Hitachi

- NB5000

JEOL USA

- JSM-7600F

- MultiBeam:JIB-4500/4600F

Oxford Instruments

www.oxford-instruments.com

p. 15

NanoAnalysis

- X-Max

- INCA Energy

Particle Technology Labs

Seron Technologies Inc.

- AIS $2100 \mathrm{C}$

- AIS 2200

- AIS $2300 \mathrm{C}$

Specs Surface Nano

Analysis $\mathrm{GmbH}$

- Aberration corrected low energy electron microscopy (LEEM)

- Photoelectron energy microscopy (PEEM) systems

Ted Pella, Inc. www.tedpella.com

- High resolution FE-SEM Sputter Coaters

Tescan USA Inc.

www.tescan.com

p. 16 


\section{Product and Services Index}

\section{A Guide to Microscopy Products}

\section{Scanning Probe Microscopes (SPMs)}

Bruker Nano

- N8 NEOS

Hysitron, Inc.

- Triboscope TS 75

ION-TOF

- hr-MFM

Nanofactory Instruments AB www.nanofactory.com

- In situ TEM Systems

Nanonics Imaging Ltd.

- The HydraTM

Nanosurf AG

- Saphyr

Specs Surface Nano

Analysis $\mathrm{GmbH}$

- UHV system for ultra-low temperatures: JT-SPM

Veeco Instruments, Inc.

- MultiMode 8 SPM

www.veeco.com

\section{Scanning Transmission Electron Microscopes (STEMs)}

DELONG America

- LVEM5 Benchtop TEM/SEM/STEM

FEI

www.fei.com

- Titan G2 60-300

\section{Scanning Tunneling Microscopes (STMs)}

A.P.E. Research SRL

www.aperesearch.com

- PA-STM

Bruker Nano

- N8 NEOS

www.bruker-nano.com

p. 10

Nanosurf AG

- easyScan 2 STM

Specs Surface Nano

Analysis $\mathrm{GmbH}$

- UHV system for variable temperatures: SPM 150 Aarhus

\section{Scintillators}

M.E. Taylor Engineering, Inc. www.semsupplies.com

- Scintillators

- Light Pipes

\section{SEM Accessories}

Advanced Microscopy

Techniques Corp.

Bruker Nano

- QUANTAX

- QUANTAX CrystAlign

Gatan, Inc.

- Gatan SmartEBIC

Hysitron, Inc.

- SEM Picolndenter PI 85

IXRF Systems, Inc.

p. 10

p. 13

p. 14

p. 14

www.nanosurf.com

www.specs.com

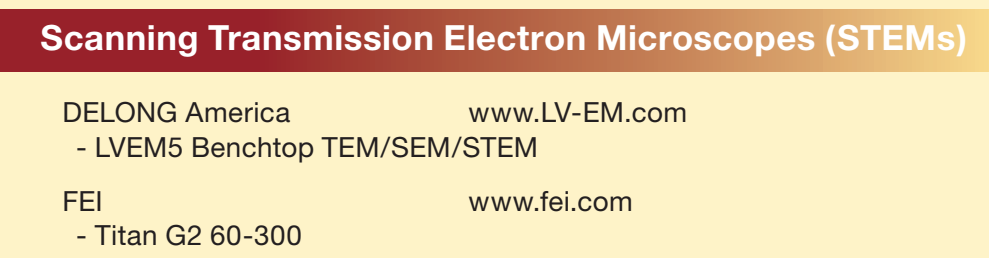




\section{Product and Services Index}

\section{A Guide to Microscopy Products}

\section{Service and Repair}

\section{MAX Detector Repair}

Group LLC

- Liquid Nitrogen X-Ray Detector Repair+Service

SIA (Scientific Instruments www.sia-cam.com

and Applications)

\section{Service Laboratories}

Alliance Technologies

McCrone Associates

Particle Technology Labs

PolyInsight LLC

- Microscopy Services

\section{Shutters}

Sutter Instrument

www.sutter.com

p. 15

- SmartShutter ${ }^{\mathrm{TM}}$

\section{Shuttering}

Sutter Instrument

www.sutter.com

p. 15

\section{Slides and Coverslips}

Globe Scientific Inc.

www.globescientific.com

- Cover Glass \& Slides

ibidi, LLC

www.ibidi.com

- U-slides/U-dishes

\section{Small Dual Beam}

FEI

www.fei.com

- Helios Nanolab

\section{Small Tools}

Minitool, Inc.

- Minitool MicroTools

www.minitoollnc.com

p. 14

- Minitool Precision MicroTools

\section{Software for Microanalysis}

4pi Analysis, Inc.

Allied High Tech Products

www.4pi.com

p. 10

Amerinex Applied Imaging www.alliedhightech.com

p. 10

- Aphelion

Chemlmage Corporation

- Chemlmage Xpert

\section{EDAX Inc.}

800-535-edax

\section{www.edax.com}

\section{See Company Profile}

- TEAM ${ }^{\mathrm{TM}}$ (EDS)

- Genesis (EDS)

- OIM $^{\mathrm{TM}}($ EBSD)

www.chemimage.com
Oxford Instruments

NanoAnalysis

- INCAEnergy

- INCAWave

\section{Software for Microscopy}

Amerinex Applied Imaging www.amerineximaging.com - Aphelion

HREM Research Inc. - HREM Stimulation Suite (xHREM)

\section{Specimen Holders}

Applied Scientific www.ASlimaging.com

Instrumentation (ASI)

- Stage Inserts

Gatan, Inc.

www.gatan.com

- Gatan 910 Multi-Specimen Single Tilt Cryo Transfer Holder

Nanofactory Instruments AB www.nanofactory.com

- In situ TEM Systems

Tousimis Research

www.tousimis.com

p. 16

\section{Corporation}

- Sample Holders

\section{Specimen Preparation Equipment}

Buehler www.buehler.com

- \#49-7251 EcoMet 250 Grinder-Polisher with Touchscreen

Denton Vacuum, LLC www.dantonvacuum.com - Desk V

Gatan, Inc.

www.gatan.com

- Gatan 601 TPC-Tool Ultrasonic Cutter

JEOL USA www.jeolusa.com

- CP/IB-09010 Cross Section Polisher

Leica Microsystems www.leica-microsystems.com - The entire range for materials and biological applications

Minitool, Inc. www.minitoollnc.com

- Carbide Micro Cutting Tools

- Minitool Precision MicroTools

Ted Pella, Inc.

www.tedpella.com

- Cressington Sputter and Carbon Coaters

\section{Specimen Preparation Supplies}

Dune Sciences, Inc. - SMART GRIDS Tack Strips

Delaware Diamond Knives www.dunesciences.com

- Diamond Knives

- Tungsten Carbide Knives

- Sapphire Steel

Globe Scientific Inc. - Microscope Slides

Omniprobe, Inc. - SSTTM

Ted Pella, Inc. www.tedpella.com 


\section{Product and Services Index}

\section{A Guide to Microscopy Products}

\section{Spectrometers}

Oxford Instruments NanoAnalysis

- X-Max

\section{Spectrometers (light)}

CRAIC Technologies, Inc. www.microspectra.com

- 20/20 PV UV-visible-NIR Microspectrophotometer

- QDI 302 Microscope Spectrometer

Renishaw, Inc.

www.renishaw.com

- Raman Microscope System

\section{Spectrometers (X-ray)}

\section{Bruker Nano}

- QUANTAX

- S2 PICOFOX

e2v Scientific Instruments www.e2vsi.com

- siriusSD (SDD) and Sirius (SiLi) detectors

\section{Spinning Disk Confocal Microscopy}

Lambert Instruments www.lambert-instruments.com

- LIFA for confocal and for TIRF microscopy

\section{Stage Automation}

\section{Applied Scientific}

Instrumentation (ASI)

- ASI XY and Z stages

\section{Stains}

\section{Electron Microscopy \\ Sciences}

215-412-8400

See Company Profile

See Our Products

www.ASlimaging.com

\section{Standards for Microanalysis}

\section{APPLIED IMAGE Inc.}

- APPLIED IAM, Accu-Place standards

Dune Sciences, Inc.

- SMART Gold Kits

\section{Stereo 3D Imaging and Measurement}

Energy Beam Sciences, Inc. www.ebsstore.com

- Alicona MeX 5.1 Imaging \& Measurement Software

\section{Evex Inc.}

See Company Profile
609-252-9192

www.evex.com p. 4

p. 10 p. 10 


\section{Product and Services Index}

\section{A Guide to Microscopy Products}

\section{TEM Accessories}

Advanced Microscopy

Techniques Corp.

Bruker Nano

- QUANTAX

Electron Microscopy

Sciences

215-412-8400

www.emsdiasum.com

See Company Profile

See Our Products

Hysitron, Inc.

- TEM Picolndenter PI 95

Ladd Research Industries

- Parts and Supplies

Nanofactory Instruments AB www.nanofactory.com

- In situ TEM Systems

SIA (Scientific Instruments

and Applications)

www.sia-cam.com

SPI Supplies

www.2spi.com

- Grids

Ted Pella, Inc.

www.tedpella.com

- PELCO Silicon Nitride Support Films

- TEM Grids and Support Films

Tousimis Research

www.tousimis.com

Corporation

- TEM Laboratory Supplies

www.laddresearch.com

Minitool, Inc.

www.minitoollnc.com

- Minitool Precision MicroTools

\section{Tools}

\section{Minitool, Inc.}

- Minitool Stainless Steel Micro Tools

www.minitoollnc.com

\section{Total Internal Reflection Microscopy (TIRF)}

p. 4

p. 10

p. 13

\section{Testing Equipment}

\section{LECO Corporation}

www.leco.com

Spectronics Corporation

www.spectroline.com

- AccuMAX'M Series of digital radiometers/photometers

Zemetrics, Inc.

www.zemetrics.com

- ZeMapper 3D Metrology System

\section{Thin Film Measurement}

Oxford Instruments

NanoAnalysis

- ThinFilmID

\section{Tissue Processors}

\section{Electron Microscopy \\ Sciences}

215-412-8400

www.emsdiasum.com

\section{See Company Profile}

See Our Products www.oxford-instruments.com p. 15

\section{Tomography}
Olympus America, Inc.
- Cell^TIRF
www.olympusamerica.com
p. 14

Olympus America, Inc. $\quad 800-446-5967$

www.olympusamerica.com

Olympus Soft Imaging $\quad$ +4925179800 0

Solutions

www.soft-imaging.net

See Company Profile

p. 14

\section{Transmission Electron Microscopes (TEMs)}

Carl Zeiss SMT Inc.

- LIBRA $^{\circledR} 120 / 200$ KV

DELONG America

- LVEM5 Benchtop TEM/SEM/STEM

FEI

- Titan G2 60-300

- TECNA: Osiris

Hitachi

- NB5000

www.hitachi-hta.com

p. 12

Hysitron, Inc.

- TEM Picolndenter PI 95

www.hysitron.com

p. 13

Oxford Instruments

www.oxford-instruments.com

p. 15

NanoAnalysis

- X-Max

- INCA Energy

Ted Pella, Inc.

www.tedpella.com

p. 16

- The PELCO BioWave Pro Microwave Tissue Processor

- PELCO easiGlow Grid Cleaning System

\section{Tweezers}

Electron Microscopy

Sciences

See Company Profile

See Our Products
215-412-8400

www.emsdiasum.com

p. 4

p. 10
SPI Supplies

- Tweezers for Research and Science

Ted Pella, Inc.

www.tedpella.com

p. 16 


\section{Product and Services Index}

\section{A Guide to Microscopy Products}

\section{Vacuum}

\section{Ultramicrotomes}

Leica Microsystems

www.leica-microsystems.com

- Leica EM UC7 with optional EM FC7 cryo attachment

Micro Star Technologies www.microstartech.com

\section{Ultraviolet Microscope}

CRAIC Technologies, Inc. www.microspectra.com

- UVM-1 UV-visible-NIR Microscope

- UVM-2 UV-visible-NIR Microscope

\section{Upright Microscope}

Olympus America, Inc.

www.olympusamerica.com

p. 14

- $B X^{\circledR}$ series microscopes

Olympus America, Inc.

800-446-5967

www.olympusamerica.com

\section{Olympus Soft Imaging} Solutions

+49251798000

www.soft-imaging.net

\section{See Company Profile}

See Our Products

\section{Used Equipment}

\section{SEMTech Solutions, Inc. 978-663-9822 (235)}

www.semtechsolutions.com

\section{See Company Profile}

p. 8

- Used and Refurbished SEMs

Pfeiffer Vacuum

- Gauges

\section{Vacuum Equipment}

Capovani Brothers Inc.

www.capovani.com

- Varian Turbomolecular Vacuum Pumps

Denton Vacuum, LLC

www.dantonvacuum.com

- Explorer

Duniway Stockroom Corp.

- Ion Pumps

- Vacuum Gauges

\section{Electron Microscopy \\ Sciences}

215-412-8400

See Company Profile

See Our Products

\section{www.emsdiasum.com}

p. 4

p. 10
Kurt J. Lesker Co.

www.lesker.com
Pfeiffer Vacuum

Synergy Vacuum, Inc. www.pfeiffer-vacuum.com

- ISP 50 Scroll Vacuum Pump 2.8 cfm 100 mTorr

- ISP 90 Scroll Vacuum Pump 3.8 cfm 38 mTorr

- ISP 250c Scroll Vacuum Pump 10.6 cfm 12 mTorr

- ISP 500c Scroll Vacuum Pump 21.2 cfm 7.5 mTorr

- ISP 1000 Scroll Vacuum Pump 42.4 cfm 7.5 mTorr

Ted Pella, Inc. $\quad$ www.tedpella.com

- Vacuum Pumps and Gauges

\section{Vacuum Evaporators}

Denton Vacuum, LLC

- Bench Top Turbo IV

Kurt J. Lesker Co. www.lesker.com

- Thermal evaporation sources

Ladd Research Industries www.laddresearch.com

- LADD Vacuum, Sputtering Systems

Ted Pella, Inc.

www.tedpella.com

p. 16

- Cressington 308R-EM

\section{Vacuum Oils}

Kurt J. Lesker Co.

www.lesker.com

- Vacuum pump oils

SPI Supplies

www.2spi.com

- Vacuum Greases

Variable-Pressure Scanning Electron Microscopes

Tescan USA Inc.

www.tescan.com

p. 16

- VEGA-3

\section{Vibration Isolation Systems}

Technical Manufacturing

www.techmfg.com

p. 15

\section{Corporation}

- STACIS ${ }^{\circledR}$ iX SEM-Base ${ }^{\text {TM }}$ Floor Platform

- STACIS ${ }^{\circledR}$ Active Piezoelectric Vibration Cancellation

- STACIS ${ }^{\circledR}$ iX LaserTable-Base ${ }^{\mathrm{TM}}$

- STACIS ${ }^{\circledR}$ iX Stage-Base ${ }^{\mathrm{TM}}$ Vibration Cancellation

\section{Vibratomes}

\section{Electron Microscopy \\ Sciences}

See Company Profile

See Our Products

\section{Video Microscopes}

Applied Scientific

Instrumentation (ASI)

- VTS-2000

- VM-2000

- MIM
215-412-8400

www.emsdiasum.com

p. 4

p. 10
- Vacuum components 


\section{Product and Services Index}

\section{A Guide to Microscopy Products}

\section{Video Microscopes (continued)}

CrEST srl

www.crestopt.com

p. 10

Keyence Corporation

www.keyence.com

- VW-6000 High-Speed Microscope

Mitutoyo America Corp. www.mitutoyo.com

\section{Water Chillers}

Capovani Brothers Inc.

www.capovani.com

- Thermo Scientific Neslab Baths/Circulators

\section{Wavelength Dispersive Super Detectors}

Advanced MicroBeam, Inc. www.advancedmicrobeam.com

\section{Wavelength Dispersive X-ray Spectrometry (WDS)}

EDAX Inc.

800-535-edax www.edax.com

See Company Profile

- LEXS Spectrometer

- TEXS HP Spectrometer

Oxford Instruments NanoAnalysis

- INCAWave

\section{Wavelength Switchers}

Sutter Instrument

www.sutter.com

p. 15

- Lambda DG-4 / DG-5 Plus

\section{Xenon Lamps}

Hamamatsu Corporation

www.sales.hamamatsu.com

- Xenon Lamps

Sutter Instrument

www.sutter.com

p. 15

- Lambda LS

\section{X-ray Analysis Equipment}

4pi Analysis, Inc.

www.4pi.com

p. 10

Charles Supper Company, Inc. www.charles-supper.com

- Goniometer Heads, Thin-Walled Capillary Tubes
EDAX Inc.

800-535-edax

www.edax.com

\section{See Company Profile}

- TEAM ${ }^{\mathrm{TM}}$ Analysis System (EDS)

- Genesis Analysis System (EDS)

- OIM ${ }^{\mathrm{TM}}$ Analysis System (EBSD)

- Pegasus Analysis System (EDS + EBSD)

- Neptune Analysis System (EDS + WDS)

- Trident Analysis System (EDS, EBSD, + WDS)

- Orbis Micro-XRF Analysis System
Evex Inc.

See Company Profile

IXRF Systems, Inc.

See Company Profile

See Our Products

Oxford Instruments

NanoAnalysis

- X-Max

- INCAEnergy

- INCAWave

SII Nanotechnology USA, Inc. www.siintusa.com - Spectrometers

Tousimis Research

Corporation

- X-Ray Reference Standards

Xradia, Inc.

- X-ray Microscopes for Synchrotrons

281-286-6485
609-252-9192

www.evex.com www.ixrfsystems.com

p. 6

p. 13

www.oxford-instruments.com

p. 15

\section{X-Ray Photoelectron Spectrometers/Microscopes}

Kratos Analytical, Inc.

www.kratos.com

- X-Ray Photoelectron Spectrometers (XPS)

\section{$\mu-X R F$ Spectrometry}

Bruker Nano

www.bruker-nano.com

p. 10

- M1 Mistral

- ARTAX

- M4 Tornado 